\title{
A Fixed Point Theorem of the Hardy and Rogers Kind Endowed with Multiplicative Cone-C Class Functions
}

(In Memory of my Grandpa)

\section{Clement Boateng Ampadu}

31 Carrolton Road, Boston, MA 02132-6303, USA; e-mail: drampadu@ hotmail.com

\begin{abstract}
In this paper we introduce the multiplicative version of cone-C class functions [1], and obtain some contraction mapping theorems of the Hardy and Rogers kind in multiplicative cone metric space endowed with such functions. Further, we propose some open problems that are publishable in nature.
\end{abstract}

\section{Introduction and Preliminaries}

Definition 1.1 [2]. Let $E$ be a real Banach space. A subset $P$ of $E$ is called a multiplicative cone iff

(a) $P$ is closed, nonempty and $P \neq \varnothing$

(b) $x^{a} \cdot y^{b} \in P$ for all $x, y \in P$ and nonnegative real numbers $a, b$

(c) $P \cap\left(\frac{1}{P}\right)=\{1\}$.

Definition 1.2 [2]. Given a multiplicative cone $P \subset E$, we define a partial ordering $\leq$ with respect to $P$ by $x \leq y$ iff $\frac{y}{x} \in P$.

Received: March 26, 2019; Accepted: May 12, 2019

2010 Mathematics Subject Classification: 47H10.

Keywords and phrases: multiplicative C-class functions, cone-C class functions, fixed point theorems.

Copyright (C) 2019 Clement Boateng Ampadu. This is an open access article distributed under the Creative Commons Attribution License, which permits unrestricted use, distribution, and reproduction in any medium, provided the original work is properly cited. 
Notation 1.3 [2]. We write $x<y$ to indicate $x \leq y$ but $x \neq y$, while $x<<$ will stand for $\frac{y}{x} \in \operatorname{int}(P)$, where $\operatorname{int}(P)$ denotes the interior of $P$.

Definition 1.4 [2]. We say the multiplicative cone $P$ is multiplicative normal, if there exists a constant $K>0$ such that for all $x, y \in E, 1 \leq x \leq y$ implies

$$
\|x\| \leq\|y\|^{K} \text {. }
$$

The least positive number satisfying the above is called the multiplicative constant of $P$. Here $\|\cdot\|$ denotes a multiplicative norm.

Definition 1.5 [2]. If $\left\{x_{n}\right\}$ is a sequence such that

$$
x_{1} \leq x_{2} \leq \cdots \leq x_{n} \leq \cdots \leq y
$$

for some $y \in E$, implies there is $x \in E$ such that

$$
\left\|x_{n}-x\right\| \rightarrow 1 \quad(n \rightarrow \infty),
$$

then the multiplicative cone $P$ is called multiplicative regular. Again $\|\cdot\|$ denotes a multiplicative norm.

Remark 1.6 [2]. In this paper, we assume $E$ is a Banach space, $P$ is a multiplicative cone in $E$ with $\operatorname{int}(P) \neq \varnothing$ and $\leq$ is a partial ordering with respect to $P$.

Definition 1.7 [2]. Let $X$ be a nonempty set. Suppose the mapping $m: X \times X \mapsto E$ satisfies

(a) $1<m(x, y)$ for all $x, y \in X$ and $m(x, y)=1$ iff $x=y$,

(b) $m(x, y)=m(y, x)$ for all $x, y \in X$,

(c) $m(x, y) \leq m(x, z) \cdot m(z, y)$ for all $x, y, z \in X$.

Then we say $m$ is a multiplicative cone metric on $X$, and call $(X, m)$ a multiplicative cone metric space.

Example 1.8. Let $E=\mathbb{R}^{2}, \quad P=\{(x, y) \in E \mid x, y \geq 1\} \subset \mathbb{R}^{2}, \quad X=\mathbb{R}, \quad$ and $m: X \times X \mapsto E$ be such that $m(x, y)=\left(a^{|x-y|}, a^{\alpha|x-y|}\right)$, where $\alpha \geq 0$ is a constant, and $a>1$ is a constant. Then $(X, m)$ is a multiplicative cone metric space. 
Definition 1.9 [2]. Let $(X, m)$ be a multiplicative cone metric space. Let $\left\{x_{n}\right\}$ be a sequence in $X$ and $x \in X$. If for every $c \in E$ with $1 \ll c$, there is $N$ such that for all $n>N, \quad m\left(x_{n}, x\right) \ll c$, then we say $\left\{x_{n}\right\}$ is multiplicative convergent, and multiplicative converges to $x$, and $x$ is the limit of $\left\{x_{n}\right\}$. In particular, we write

$$
\lim _{n \rightarrow \infty} x_{n}=x \text { or } x_{n} \rightarrow x(n \rightarrow \infty) \text {. }
$$

Definition 1.10 [2]. Let $(X, m)$ be a multiplicative cone metric space, $\left\{x_{n}\right\}$ be a sequence in $X$. If for any $c \in E$ with $1<<c$, there is $N$ such that for all $n, k>N$, $m\left(x_{n}, x_{k}\right)<c$, then we say $\left\{x_{n}\right\}$ is a multiplicative Cauchy sequence in $X$.

Definition 1.11 [2]. Let $(X, m)$ be a multiplicative cone metric space. If every multiplicative Cauchy sequence is multiplicative convergent in $X$, then we say $X$ is a complete multiplicative cone metric space.

\section{Main Results}

In the sequel we will need the following new ideas. The first concept is inspired by the idea of altering distance function, and for example see [1].

Definition 2.1. A function $\psi: P \mapsto P$ is called a modified altering distance function if the following properties hold:

(a) $\psi$ is nonincreasing and continuous,

(b) $\psi(t)=1$ iff $t=1$.

Inspired by the concept of ultra ultering distance function, and for example see [1], we introduce the following

Definition 2.2. A function $\varphi: P \mapsto P$ is called a modified ultra ultering distance function if the following properties are satisfied

(a) $\varphi(t)>1$ for all $t>1$,

(b) $\varphi(1) \geq 1$.

Inspired by the concept of multiplicative C-class functions [3], we introduce its version in multiplicative cone metric space as follows 
Definition 2.3. A mapping $F^{*}: P^{2} \mapsto P$ is called a multiplicative cone $C$-class function if it is continuous and satisfies the following axioms

(a) $F^{*}(s, t) \leq s$,

(b) $F^{*}(s, t)=s$ implies either $s=1$ or $t=1$, for all $s, t \in P$.

Notation 2.4. The set of all multiplicative cone C-class functions will be denoted by $C^{*}$.

Example 2.5. The following are elements of $C^{*}$.

(a) $F^{*}(s, t)=\frac{s}{t}$,

(b) $F^{*}(s, t)=s^{k}$, where $0<k<1$,

(c) $F^{*}(s, t)=\beta(s)^{s}$, where for some $a>1, \beta:[1, \infty) \mapsto[1, a)$,

(d) $F^{*}(s, t)=\Psi(s)$, where $\Psi: P \mapsto P, \Psi(1)=1, \Psi(s)>1$ for all $s \in P$ with $s \neq 1$, and $\Psi(s) \leq s$ for all $s \in P$,

(e) $F^{*}(s, t)=\frac{s}{\varphi(s)}$, where $\varphi:[1, \infty) \mapsto[1, \infty)$ is a continuous function such that $\varphi(t)=1$ iff $t=1$,

(f) $F^{*}(s, t)=\frac{s}{h(s, t)}$, where $h:[1, \infty)^{2} \mapsto[1, \infty)$ is a continuous function such that $h(s, t)=1$ iff $t=1$ for all $t, s>1$,

(g) $F^{*}(s, t)=\varphi(s), \quad F^{*}(s, t)=s$ implies $s=1$, where $\varphi:[1, \infty) \mapsto[1, \infty)$ is an upper semi-continuous function such that $\varphi(1)=1$ and $\varphi(t)<t$ for all $t>1$.

Inspired by the inequality in the Hardy and Rogers mapping theorem [4], we introduce the following

Definition 2.6. Let $(X, m)$ be a multiplicative cone metric space, $\psi$ be a modified altering distance function, $\varphi$ be a modified ultra ultering distance function, and 
$F^{*} \in C^{*}$. We say a self map $T$ of $(X, m)$ is a $\psi-\varphi-C^{*}$ multiplicative operator of the Hardy and Rogers type if it satisfies the following inequality for all $x, y \in X$, where $0 \leq k<\frac{1}{5}$

$$
\begin{array}{r}
\psi(m(T x, T y)) \leq F^{*}\left(\psi \left(\left((m(x, y) \cdot m(x, T x) \cdot m(y, T y) \cdot m(x, T y) \cdot m(y, T x))^{k}\right),\right.\right. \\
\varphi\left(\left((m(x, y) \cdot m(x, T x) \cdot m(y, T y) \cdot m(x, T y) \cdot m(y, T x))^{k}\right)\right) .
\end{array}
$$

Inspired by the inequality in the Reich mapping theorem [5], we introduce the following

Definition 2.7. Let $(X, m)$ be a multiplicative cone metric space, $\psi$ be a modified altering distance function, $\varphi$ be a modified ultra ultering distance function, and $F^{*} \in C^{*}$. We say a self map $T$ of $(X, m)$ is a $\psi-\varphi-C^{*}$ multiplicative operator of the Reich type if it satisfies the following inequality for all $x, y \in X$, where $0 \leq k<\frac{1}{3}$,

$$
\begin{array}{r}
\psi(m(T x, T y)) \leq F^{*}\left(\psi \left(\left((m(x, y) \cdot m(x, T x) \cdot m(y, T y))^{k}\right),\right.\right. \\
\varphi\left(\left((m(x, y) \cdot m(x, T x) \cdot m(y, T y))^{k}\right)\right) .
\end{array}
$$

Inspired by the inequality in the Kannan mapping theorem [6], we introduce the following

Definition 2.8. Let $(X, m)$ be a multiplicative cone metric space, $\psi$ be a modified altering distance function, $\varphi$ be a modified ultra ultering distance function, and $F^{*} \in C^{*}$. We say a self map $T$ of $(X, m)$ is a $\psi-\varphi-C^{*}$ multiplicative operator of the Kannan type if it satisfies the following inequality for all $x, y \in X$, where $0 \leq k<\frac{1}{2}$

$$
\psi(m(T x, T y)) \leq F^{*}\left(\psi \left(\left((m(x, T x) \cdot m(y, T y))^{k}\right), \varphi\left(\left((m(x, T x) \cdot m(y, T y))^{k}\right)\right) .\right.\right.
$$

Inspired by the inequality in the Chatterjee mapping theorem [7], we introduce the following 
Definition 2.9. Let $(X, m)$ be a multiplicative cone metric space, $\psi$ be a modified altering distance function, $\varphi$ be a modified ultra ultering distance function, and $F^{*} \in C^{*}$. We say a self map $T$ of $(X, m)$ is a $\psi-\varphi-C^{*}$ multiplicative operator of the Chatterjee type if it satisfies the following inequality for all $x, y \in X$, where $0 \leq k<\frac{1}{2}$,

$$
\psi(m(T x, T y)) \leq F^{*}\left(\psi \left(\left((m(x, T y) \cdot m(y, T x))^{k}\right), \varphi\left(\left((m(x, T y) \cdot m(y, T x))^{k}\right)\right) .\right.\right.
$$

Inspired by the inequality in the Banach mapping theorem [8], we introduce the following

Definition 2.10. Let $(X, m)$ be a multiplicative cone metric space, $\psi$ be a modified altering distance function, $\varphi$ be a modified ultra ultering distance function, and $F^{*} \in C^{*}$. We say a self map $T$ of $(X, m)$ is a $\psi-\varphi-C^{*}$ multiplicative operator of the Banach type if it satisfies the following inequality for all $x, y \in X$, where $0 \leq k<1$,

$$
\psi(m(T x, T y)) \leq F^{*}\left(\psi \left(\left((m(x, y))^{k}\right), \varphi\left(\left((m(x, y))^{k}\right)\right) .\right.\right.
$$

\subsection{A fixed point theorem of the Hardy and Rogers kind}

Theorem 2.11. Let $(X, m)$ be a multiplicative cone metric space, and the self map $T$ of $(X, m)$ be a $\psi-\varphi-C^{*}$ multiplicative operator of the Hardy and Rogers type. The fixed point of $T$ is unique, provided that $(X, m)$ is a complete multiplicative cone metric space.

Proof. Define the sequence $\left\{y_{n}\right\}$ by $y_{n+1}=T y_{n}$ and observe we have the following

$$
\begin{aligned}
& \psi\left(m\left(y_{n+1}, y_{n+2}\right)\right) \\
= & \psi\left(m\left(T y_{n}, T y_{n+1}\right)\right) \\
\leq & F^{*}\left\{\psi\left(\left(m\left(y_{n}, y_{n+1}\right) \cdot m\left(y_{n}, T y_{n}\right) \cdot m\left(y_{n+1}, T y_{n+1}\right) \cdot m\left(y_{n}, T y_{n+1}\right) \cdot m\left(y_{n+1}, T y_{n}\right)\right)^{k}\right),\right. \\
& \left.\quad \varphi\left(\left(m\left(y_{n}, y_{n+1}\right) \cdot m\left(y_{n}, T y_{n}\right) \cdot m\left(y_{n+1}, T y_{n+1}\right) \cdot m\left(y_{n}, T y_{n+1}\right) \cdot m\left(y_{n+1}, T y_{n}\right)\right)^{k}\right)\right\}
\end{aligned}
$$




$$
\begin{aligned}
& =F^{*}\left\{\psi\left(\left(m\left(y_{n}, y_{n+1}\right) \cdot m\left(y_{n}, y_{n+1}\right) \cdot m\left(y_{n+1}, y_{n+2}\right) \cdot m\left(y_{n}, y_{n+2}\right) \cdot m\left(y_{n+1}, y_{n+1}\right)\right)^{k}\right)\right. \text {, } \\
& \left.\varphi\left(\left(m\left(y_{n}, y_{n+1}\right) \cdot m\left(y_{n}, y_{n+1}\right) \cdot m\left(y_{n+1}, y_{n+2}\right) \cdot m\left(y_{n}, y_{n+2}\right) \cdot m\left(y_{n+1}, y_{n+1}\right)\right)^{k}\right)\right\} \\
& =F^{*}\left\{\psi\left(\left(m\left(y_{n}, y_{n+1}\right)^{2} \cdot m\left(y_{n+1}, y_{n+2}\right) \cdot m\left(y_{n}, y_{n+2}\right)\right)^{k}\right),\right. \\
& \left.\varphi\left(\left(m\left(y_{n}, y_{n+1}\right)^{2} \cdot m\left(y_{n+1}, y_{n+2}\right) \cdot m\left(y_{n}, y_{n+2}\right)\right)^{k}\right)\right\} \\
& \leq F^{*}\left\{\psi\left(\left(m\left(y_{n}, y_{n+1}\right)^{2} \cdot m\left(y_{n+1}, y_{n+2}\right) \cdot m\left(y_{n}, y_{n+1}\right) \cdot m\left(y_{n+1}, y_{n+2}\right)\right)^{k}\right),\right. \\
& \left.\varphi\left(\left(m\left(y_{n}, y_{n+1}\right)^{2} \cdot m\left(y_{n+1}, y_{n+2}\right) \cdot m\left(y_{n}, y_{n+1}\right) \cdot m\left(y_{n+1}, y_{n+2}\right)\right)^{k}\right)\right\} \\
& =F^{*}\left\{\psi\left(\left(m\left(y_{n}, y_{n+1}\right)^{3} \cdot m\left(y_{n+1}, y_{n+2}\right)^{2}\right)^{k}\right), \varphi\left(\left(m\left(y_{n}, y_{n+1}\right)^{3} \cdot m\left(y_{n+1}, y_{n+2}\right)^{2}\right)^{k}\right)\right\} \\
& \leq \psi\left(\left(m\left(y_{n}, y_{n+1}\right)^{3} \cdot m\left(y_{n+1}, y_{n+2}\right)^{2}\right)^{k}\right) .
\end{aligned}
$$

The above inequality implies that

$$
m\left(y_{n+1}, y_{n+2}\right) \leq m\left(y_{n}, y_{n+1}\right)^{3 k} \cdot m\left(y_{n+1}, y_{n+2}\right)^{2 k} \text {. }
$$

From the above we deduce that

$$
m\left(y_{n+1}, y_{n+2}\right) \leq m\left(y_{n}, y_{n+1}\right) \frac{3 k}{1-2 k}
$$

Let $h:=\frac{3 k}{1-2 k}$, then the above implies

$$
m\left(y_{n+1}, y_{n+2}\right) \leq m\left(y_{n}, y_{n+1}\right)^{h}
$$

and by induction for all $n \in \mathbb{N} \cup\{0\}$ we have $m\left(y_{n}, y_{n+1}\right) \leq m\left(y_{0}, y_{1}\right)^{h^{n}}$. Now we show that $\left\{y_{n}\right\}$ is a multiplicative Cauchy sequence. For this, let, $n, k \in \mathbb{N} \cup 0$ with $n<k$ and observe that we have the following

$$
\begin{aligned}
m\left(y_{n}, y_{k}\right) & \leq m\left(y_{n}, y_{n+1}\right) \cdots m\left(y_{k-1}, y_{k}\right) \\
& \leq m\left(y_{0}, y_{1}\right)^{h^{n}} \cdots m\left(y_{0}, y_{1}\right)^{h^{k-1}} \\
& =m\left(y_{0}, y_{1}\right)^{h^{n}+\cdots+h^{k-1}}
\end{aligned}
$$




$$
\begin{aligned}
& \leq m\left(y_{0}, y_{1}\right)^{h^{n}+h^{n+1}+\cdots} \\
& \leq m\left(y_{0}, y_{1}\right) \frac{h^{h}}{1-h}
\end{aligned}
$$

From the above, we deduce that

$$
\left\|m\left(y_{n}, y_{k}\right)\right\| \leq\left\|m\left(y_{0}, y_{1}\right)\right\|^{K} \frac{h^{n}}{1-h} .
$$

Since $h<1$, it now follows that $\lim _{n, k \rightarrow \infty} m\left(y_{n}, y_{k}\right)=1$, and hence $\left\{y_{n}\right\}$ is a multiplicative Cauchy sequence. Since $X$ is multiplicative complete, there is $y^{*} \in X$ such that $\lim _{n \rightarrow \infty} y_{n}=y^{*}$. Now we show existence of the fixed point. Observe we have the following

$$
\begin{aligned}
& \psi\left(m\left(y_{n+1}, T y^{*}\right)\right) \\
= & \psi\left(m\left(T y_{n}, T y^{*}\right)\right) \\
\leq & F^{*}\left(\psi \left(\left(\left(m\left(y_{n}, y^{*}\right) \cdot m\left(y_{n}, T y_{n}\right) \cdot m\left(y^{*}, T y^{*}\right) \cdot m\left(y_{n}, T y^{*}\right) \cdot m\left(y^{*}, T y_{n}\right)\right)^{k}\right),\right.\right. \\
& \quad \varphi\left(\left(\left(m\left(y_{n}, y^{*}\right) \cdot m\left(y_{n}, T y_{n}\right) \cdot m\left(y^{*}, T y^{*}\right) \cdot m\left(y_{n}, T y^{*}\right) \cdot m\left(y^{*}, T y_{n}\right)\right)^{k}\right)\right) \\
= & F^{*}\left(\psi \left(\left(\left(m\left(y_{n}, y^{*}\right) \cdot m\left(y_{n}, y_{n+1}\right) \cdot m\left(y^{*}, T y^{*}\right) \cdot m\left(y_{n}, T y^{*}\right) \cdot m\left(y^{*}, y_{n+1}\right)\right)^{k}\right),\right.\right. \\
& \quad \varphi\left(\left(\left(m\left(y_{n}, y^{*}\right) \cdot m\left(y_{n}, y_{n+1}\right) \cdot m\left(y^{*}, T y^{*}\right) \cdot m\left(y_{n}, T y^{*}\right) \cdot m\left(y^{*}, y_{n+1}\right)\right)^{k}\right)\right) .
\end{aligned}
$$

Taking limits in the above inequality as $n \rightarrow \infty$ we deduce the following

$$
\begin{aligned}
\psi\left(m\left(y^{*}, T y^{*}\right)\right) & \leq F^{*}\left(\psi\left(m\left(y^{*}, T y^{*}\right)^{2 k}, \varphi\left(m\left(y^{*}, T y^{*}\right)^{2 k}\right)\right)\right) \\
& \leq \psi\left(m\left(y^{*}, T y^{*}\right)^{2 k}\right) .
\end{aligned}
$$

From the above we deduce the following

$$
m\left(y^{*}, T y^{*}\right) \leq m\left(y^{*}, T y^{*}\right)^{2 k}
$$

which implies that

$$
m\left(y^{*}, T y^{*}\right)^{1-2 k} \leq 1 .
$$


Since $1-2 k \neq 0$ for any $k \in\left[0, \frac{1}{5}\right)$, we get that $m\left(y^{*}, T y^{*}\right)=1$ and hence $y^{*}=T y^{*}$.

Finally, we show the fixed point is unique. Suppose $x^{*}=T x^{*}$, but $x^{*} \neq y^{*}$. Now observe we have the following

$$
\begin{aligned}
\psi\left(m\left(x^{*}, y^{*}\right)\right) & =\psi\left(m\left(T x^{*}, T y^{*}\right)\right) \\
& \leq F^{*}\left(\psi\left(m\left(x^{*}, y^{*}\right)^{3 k}, \varphi\left(m\left(x^{*}, y^{*}\right)^{3 k}\right)\right)\right) \\
& \leq \psi\left(m\left(x^{*}, y^{*}\right)^{3 k}\right)
\end{aligned}
$$

which implies that

$$
m\left(x^{*}, y^{*}\right)^{1-3 k} \leq 1
$$

Since $1-3 k \neq 0$ for any $k \in\left[0, \frac{1}{5}\right)$, we get that $m\left(x^{*}, y^{*}\right)=1$, and hence $x^{*}=y^{*}$. So the fixed point is unique, and the proof is completed.

\subsection{A consequence}

Corollary 2.12. Let $(X, m)$ be a multiplicative cone metric space, and the self map Tof $(X, m)$ satisfy

$$
\psi(m(T x, T y)) \leq \frac{\psi\left(\left((m(x, y) \cdot m(x, T x) \cdot m(y, T y) \cdot m(x, T y) \cdot m(y, T x))^{k}\right)\right.}{\varphi\left(\left((m(x, y) \cdot m(x, T x) \cdot m(y, T y) \cdot m(x, T y) \cdot m(y, T x))^{k}\right)\right.}
$$

for all $x, y \in X$, where $\psi$ is a modified altering distance function, $\varphi$ is a modified ultra ultering distance function. The fixed point of $T$ is unique, provided that $(X, m)$ is a complete multiplicative cone metric space.

Proof. Let $F^{*}$ be given by Example 2.5(a) in Theorem 2.11.

\subsection{Open problems}

These are inspired by Definitions 2.7, 2.8, 2.9, and 2.10, and we ask the reader to prove or disprove the following 
Exercise 2.13. Let $(X, m)$ be a multiplicative cone metric space, and the self map $T$ of $(X, m)$ be a $\psi-\varphi-C^{*}$ multiplicative operator of the Reich type. The fixed point of $T$ is unique, provided that $(X, m)$ is a complete multiplicative cone metric space.

Exercise 2.14. Let $(X, m)$ be a multiplicative cone metric space, and the self map $T$ of $(X, m)$ be a $\psi-\varphi-C^{*}$ multiplicative operator of the Kannan type. The fixed point of $T$ is unique, provided that $(X, m)$ is a complete multiplicative cone metric space.

Exercise 2.15. Let $(X, m)$ be a multiplicative cone metric space, and the self map $T$ of $(X, m)$ be a $\psi-\varphi-C^{*}$ multiplicative operator of the Chatterjee type. The fixed point of $T$ is unique, provided that $(X, m)$ is a complete multiplicative cone metric space.

Exercise 2.16. Let $(X, m)$ be a multiplicative cone metric space, and the self map $T$ of $(X, m)$ be a $\psi-\varphi-C^{*}$ multiplicative operator of the Banach type. The fixed point of $T$ is unique, provided that $(X, m)$ is a complete multiplicative cone metric space.

\section{References}

[1] Arslan Hojat Ansari, Sumit Chandok, Nawab Hussin and Ljiljana Paunovic, Fixed points of $(\psi, \phi)$-weak contractions in regular cone metric spaces via new function, J. Adv. Math. Stud. 9(1) (2016), 72-82.

[2] Clement Boateng Ampadu, A coupled version of the higher-order Banach contraction principle in multiplicative cone metric space, JP Journal of Applied Mathematics, to appear. https://drive.google.com/file/d/0BwtkpMtWoUlEWVVXLS1DVjNiYWc/view

[3] Clement Ampadu and Arslan Hojat Ansari, Fixed point theorems in complete multiplicative metric spaces with application to multiplicative analogue of C-class functions, JP J. Fixed Point Theory Appl. 11(2) (2016), 113-124. https://doi.org/10.17654/FP011020113

[4] G. E. Hardy and T. D. Rogers, A generalization of a fixed point theorem of Reich, Canad. Math. Bull. 16(2) (1973), 201-206. https://doi.org/10.4153/CMB-1973-036-0

[5] S. Reich, Some remarks concerning contraction mappings, Canad. Math. Bull. 14(1) (1971), 121-124. https://doi.org/10.4153/CMB-1971-024-9 
[6] R. Kannan, Some results on fixed points - II, Amer. Math. Monthly 76 (1969), 405-408. https://doi.org/10.2307/2316437

[7] S. K. Chatterjee, Fixed point theorem, C. R. Acad. Bulgare Sci. 25 (1972), 727-730.

[8] S. Banach, Sur les opérations dans les ensembles abstraits et leur application aux équations intégrales, Fund. Math. 3 (1922), 133-181.

https://doi.org/10.4064/fm-3-1-133-181 